\title{
Tri-cresyl phosphate neuropathy in Sri Lanka: a clinical and neurophysiological study with a three year follow up
}

\author{
NIMAL SENANAYAKE \\ From the Department of Medicine, University of Peradeniya, Peradeniya, Sri Lanka
}

SUMMARY Clinical and neurophysiological findings in twenty patients intoxicated with tri-cresyl phosphate following consumption of contaminated gingili oil are reported. Marked distal axonopathy, high incidence of pyramidal tract dysfunction and minimal sensory abnormalities were the cardinal features. Despite pronounced motor weakness in the early stages, the patients showed remarkable improvement during a three year follow up.

An outbreak of acute polyneuropathy occurred in the central tea plantation area of Sri Lanka during 1977-78, affecting adolescent Tamil girls attaining menarche. The cause of the neuropathy could be attributed to tri-cresyl phosphate (TCP) which was present as a contaminant in gingili oil, the contamination presumably having occurred during transport of the oil in containers previously used for storing mineral oils. Gingili (jinjili, ginelly) oil, which is an extract of the seeds from a kind of sesame plants, is primarily a cooking oil used commonly by the Tamils. Because of its presumed nutritive value, the raw oil is given in large quantities to pubertal girls as a special diet during the immediate post-menarche period. It was this strange custom which had been responsible for the selective affliction of the adolescent Tamil girls in the present epidemic.

This paper is an analysis of the clinical and neurophysiological findings obtained in 20 patients over a three year period since poisoning. An outstanding feature was the complete recovery made by the patients, in contrast to the outcome of previously reported epidemics.

\section{Patients and methods}

All 20 patients were Indian Tamil girls aged 14-17 years, who had been in good health previously. Each patient had consumed $40-80 \mathrm{ml}$ of gingili oil daily for two weeks

Address for reprint requests: Faculty of Medicine, University of Peradeniya, Sri Lanka.

Accepted 16 June 1981 following menarche. A residual sample of oil was found to contain an isomeric mixture of TCP at a concentration of $5600 \mu \mathrm{g} / \mathrm{g}$. Accordingly, the approximate quantity of TCP ingested by each patient during the two week periods could be estimated as $2 \cdot 8-5 \cdot 6 \mathrm{~g}$. The details concerning the toxicological and epidemiological aspects of the poisoning were given in a previous paper. ${ }^{1}$

All the patients were first seen within five months of poisoning. Ten patients were available for review during the three year period. At each visit the patients were interviewed about their symptoms and a complete physical examination was performed with special reference to the nervous system. The following investigations carried out routinely at the first visit showed no significant abnormalities; erythrocyte sedimentation rate, white cell count and differential count, haemoglobin and blood film, fasting blood sugar, serum proteins, calcium, phosphate, alkaline phosphatase and lead, urine albumin, porphobilinogen and deposits, cerebrospinal fluid for proteins, cells and culture and radiography of chest and wrists.

Neurophysiological studies were carried out on 17 patients during the three year period. The studies consisted of electromyographic (EMG) examination of limb muscles and measurement of motor conduction in median and ulnar nerves. The motor conduction velocity (MCV) in the fastest conducting fibres from elbow to wrist and the terminal latency (TL) from wrist to the corresponding muscles were measured using supramaximal stimuli. Six patients who were examined during the third year, including three patients who were available for re-examination, were subjected to the following additional tests: MCV (from knee to ankle) and TL of common peroneal and tibial nerves; sensory conduction velocity (SCV) (digits to wrist) and sensory action potential (SAP) of median and ulnar nerves. All the studies were carried out using conventional electrophysiological 
techniques, at a constant room temperature of $27^{\circ} \mathrm{C}$.

\section{Results}

The symptoms appeared 14-30 days (mean: 25 days) after the first dose of gingili oil. Intense pain in calf muscles lasting 1-3 days was a constant symptom which heralded limb weakness. The latter was characterised by symmetrical involvement of the distal muscles first affecting the lower limbs and then spreading to the upper limbs. The weakness was rapidly progressive reaching its maximum in 1-3 days. At this stage the patients had marked difficulty in walking although none were bed-ridden or chairbound. Most of the patients were unable to use their hands and fingers to feed themselves in the traditional way, and they had to resort to using spoons. One patient experienced "pins and needles" sensations below mid-calf in both legs, lasting two weeks. None of the patients admitted to any gastrointestinal symptoms, sphincter disturbances or visual abnormalities.

The physical signs were primarily dependent on the time of examination during the course of the illness. The early part of the illness was dominated by signs of flaccid weakness of the distal limb muscles, more marked in the lower extremity. Bilateral foot drop was a constant feature; in $75 \%$ of patients the paralysis of dorsiflexion was complete. In some patients this was associated with mild to moderate wasting of the anterior and the peroneal compartments of the legs. Plantar flexion of feet was affected to a lesser degree. By contrast, the proximal muscles were relatively unaffected. Any involvement of the proximal muscles was limited to a slight spastic type of weakness of knee and hip flexors seen in a few patients who had additional signs of pyramidal tract dysfunction. In the upper limbs the intrinsic muscles of the hands were the worst affected. Almost all the patients had wasting of the intrinsic muscles and clawing of the hands to varying degree. The weakness was most marked in the abductor pollicis brevis. In the forearm, the muscles of the extensor compartment were affected producing wrist-drop in about $25 \%$ of patients. No significant weakness was found in the rest of the muscles. Loss of ankle jerks was a constant finding in all but one patient during the early phase of the illness. The knee jerks were decreased in $20 \%$ and exaggerated in $40 \%$ during the first five months. The other tendon reflexes were normal. The plantar response remained flexor even when the knee jerks were exaggerated. Sensory changes were present in three patients. In two patients, these were limited to decreased sensitivity to pain in the "glove and stocking" area. In another patient, joint position and vibration sense were also impaired in the distal parts of the limbs. The remaining patients showed no abnormalities on sensory testing, including two point discrimination. The rest of the nervous system and the other systems were normal.

The neurological status of the patients changed gradually during the three year period, as shown in fig 1 . The pyramidal tract signs became more apparent during the latter part of the illness. These signs were limited to mild spasticity of lower limbs and exaggeration of knee jerks. Upper limb reflexes and ankle jerks were also exaggerated in some cases. Nearly $50 \%$ of the patients showed some sign of pyramidal tract dysfunction during the course of the illness, but in none of them were the signs severe enough to produce any disability. The lower motor neurone signs began to improve and almost completely disappeared at the end of one year. The eight patients who came for follow up and four other patients had gone back to work as tea pluckers at the end of one year. Two patients ( $\mathrm{C}$ and $\mathrm{K}$ in fig 1$)$ had mild weakness of dorsiflexion of feet, but none of them had any significant symptoms. The sensory changes found in three patients had also completely disappeared at the end of one year. It is pertinent to mention that the only form of treatment they had had was physiotherapy, mainly walking exercises, for two to six weeks during the initial period in hospital.

EMG of abductor pollicis brevis, abductor digiti minimi, 1st dorsal interosseus, tibialis anterior, gastrocnemius, extensor digitorum brevis and abductor hallucis showed neuropathic changes of varying severity during the first 12 weeks. These changes included fibrillation potentials, large and polyphasic motor unit potentials and decreased interference pattern. In some muscles the electrical activity at maximum voluntary contraction was limited to single motor unit firing. In some cases no motor units could be recorded at all. Abductor pollicis brevis, extensor digitorum brevis, abductor hallucis and tibialis anterior were the earliest and the worst affected. The proximal muscles showed no significant abnormalities. In the six patients examined during the third year, the EMG was normal in all but the two patients ( $C$ and $K$ ) who had mild weakness of dorsiflexion of feet. They continued to show less marked but clear neuropathic changes in tibialis anterior, abductor hallucis and extensor digitorum brevis.

Impairment of motor conduction was most marked during the first 12 weeks. Terminal latencies were considerably prolonged during this period, as shown in fig 2 . The abnormality was most marked around the 8th week, when no motor action potentials could be evoked at all $\mathrm{b} \%$ stimulating median or 


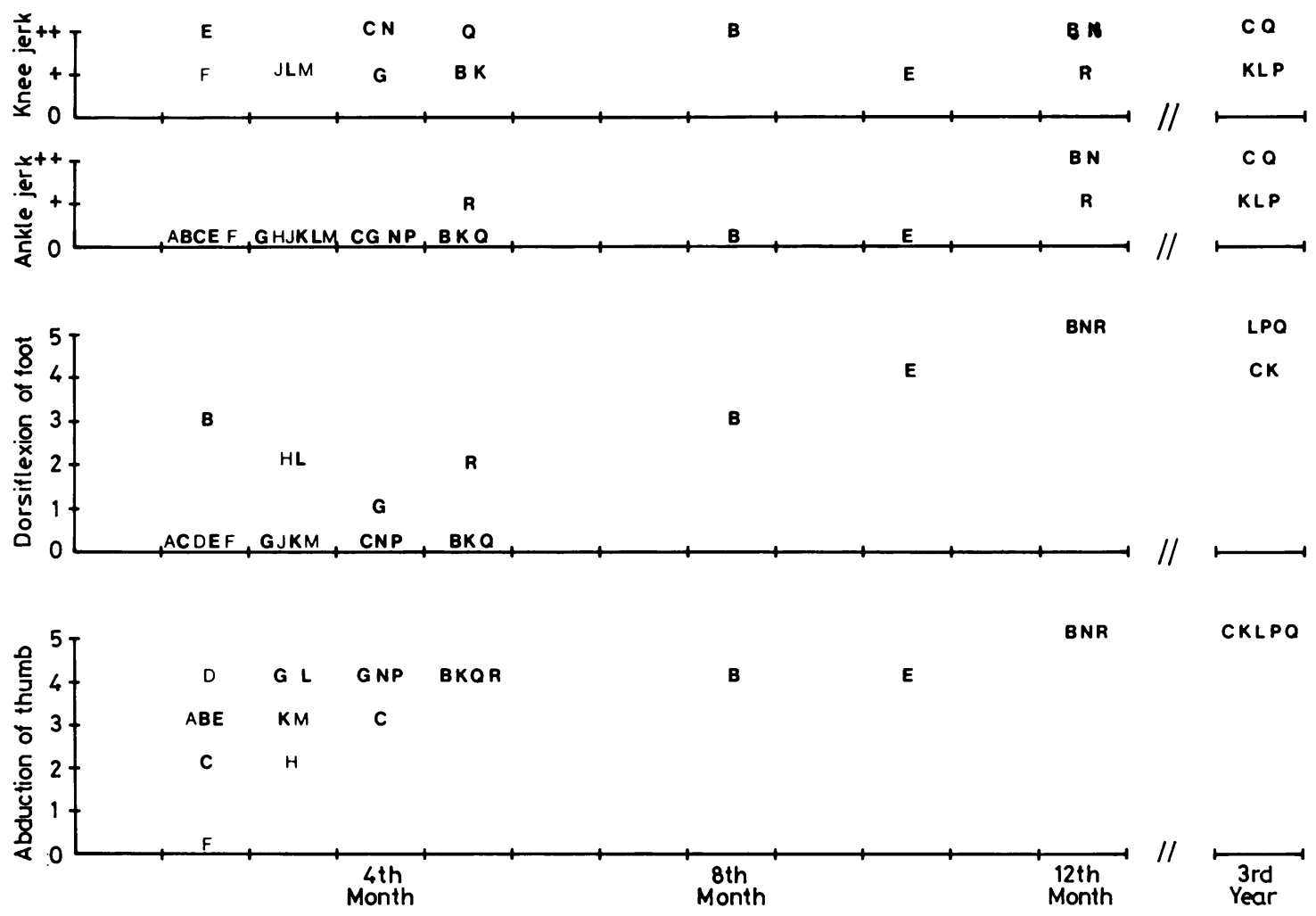

Fig 1 Assessment of the physical status during the $3 \mathrm{yr}$ period. Each letter represents a patient. Heavy lettering indicates patients who were seen more than once. Muscle power: 0-5 MRC grading. Reflexes: 0 absent, + present, ++ exaggerated.

ulnar nerves. During the third year the TLs were within normal limits.

Motor conduction velocities of median and ulnar nerves during the three year period are shown in fig 3. Two patients (A and E) showed considerable slowing of median nerve conduction in the 10th week. In the others (except in cases where stimulation of the nerves failed to evoke action potentials in the corresponding muscles) the MCV were over $40 \mathrm{~m} / \mathrm{s}$ and showed no change during the three year period.

The results of motor conduction studies on common peroneal and tibial nerves during the third year are shown in the table. In patients $C$ and $K$, stimulation of the common peroneal nerve failed to evoke motor action potentials. Patient $Q$ also showed considerable impairment of motor conduction. In others the conduction was either mildly impaired or normal. Sensory conduction studies carried out on median and ulnar nerves during the third year produced normal SCVs and SAPs.

\section{Discussion}

Neurotoxic effects of cresyl phosphates had been well recognised since the outbreak of "ginger jake paralysis" which occurred in America in the 1930s. ${ }^{2}$ In the jake paralysis as well as in a number of subsequent outbreaks, the cresyl phosphate which was responsible for the neuropathy had been considered to be the tri-ortho compound. It is of interest that in the present epidemic, the toxic contaminant found in gingili oil was an isomeric mixture of tri-cresyl phosphate (TCP) with little or no triortho-cresyl phosphate (TOCP). ${ }^{1}$ However, its exact composition is not known. TCP is derived from a mixture of cresols, principally the meta and para isomers, but containing some ortho cresol. TCP, therefore, consists of a mixture of esters, some of which will contain at least one ortho cresyl group; such esters will be neurotoxic. The overall toxic potential of TCP is known to be less than TOCP, but not insignificant. Its degree of neurotoxicity will 

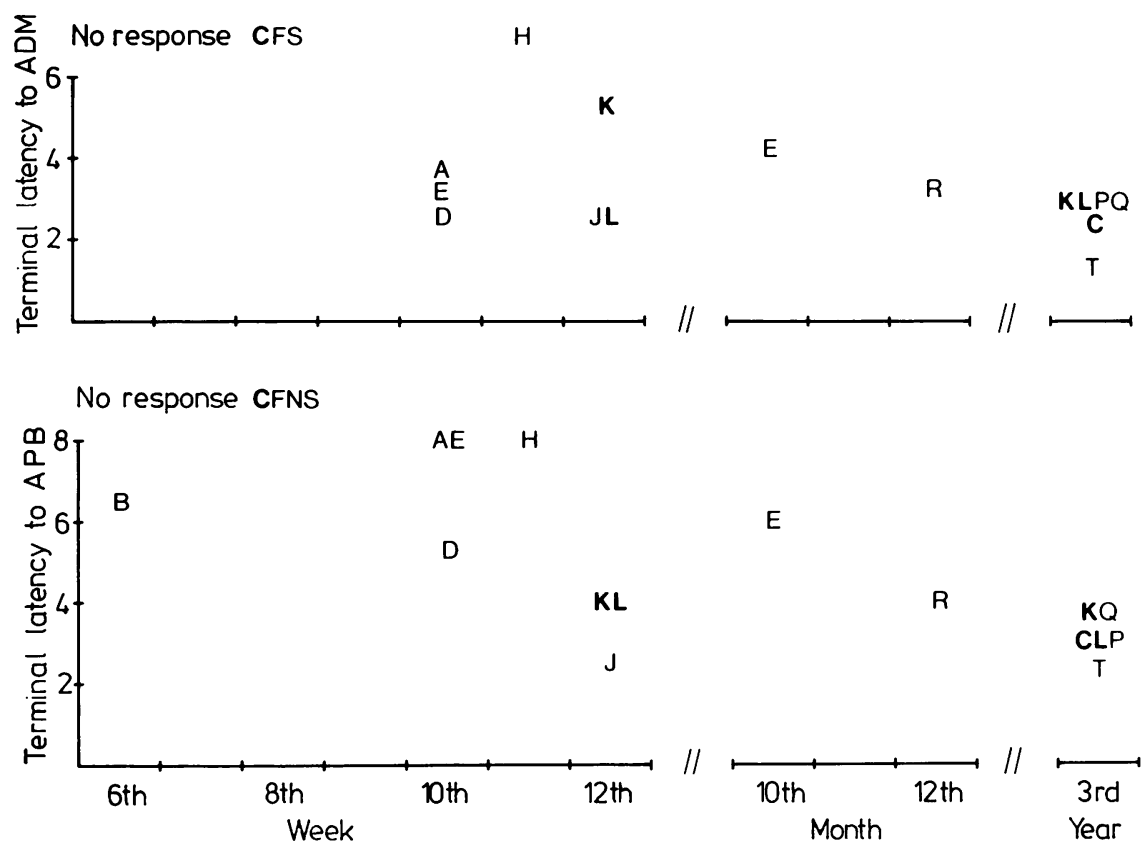

Fig 2 Terminal latencies of median and ulnar nerves. Letters refer to the same patients as in fig 2.
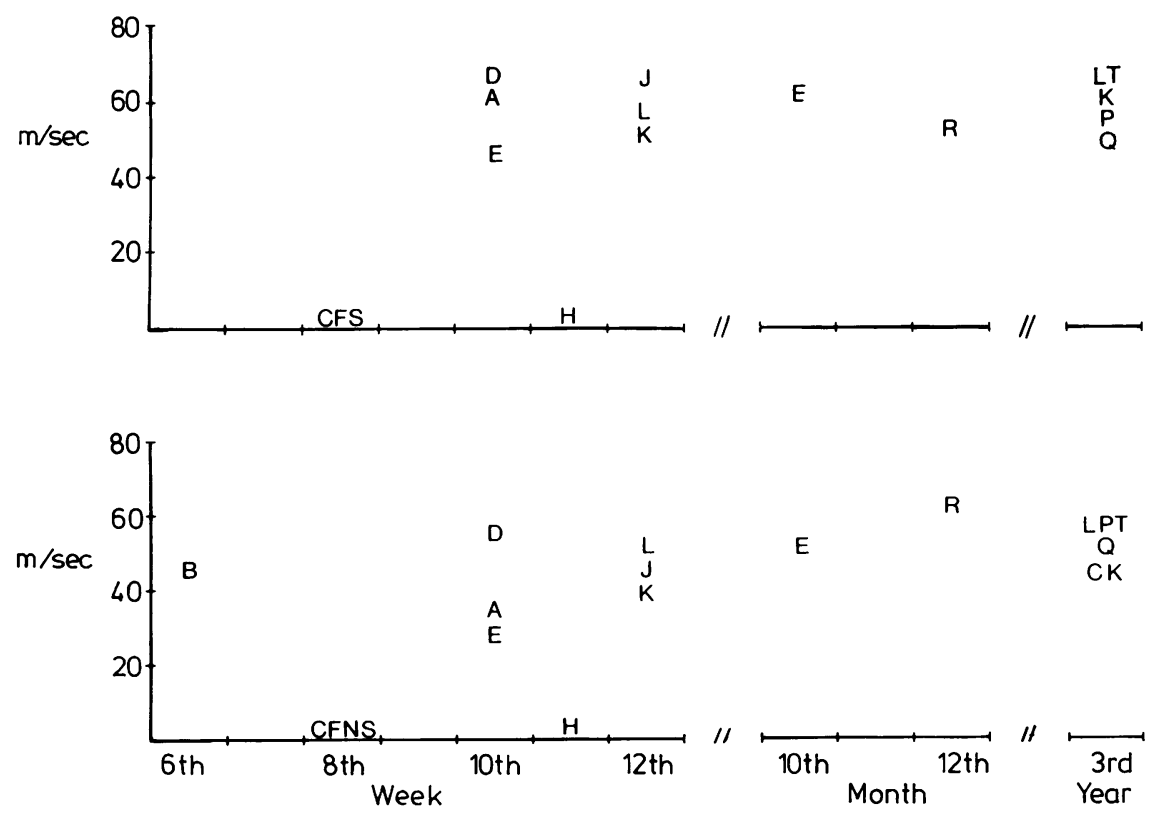

Fig 3 Motor conduction velocities (from elbow to wrist) of median nerve (below) and ulnar nerve (above). Letters refer to the same patients as in figs 1 and 2. 
Table Terminal latencies $(T L)$ and conduction velocities ( $M C V)$ (knee to ankle) in the common peroneal and tibial nerves during the third year

\begin{tabular}{|c|c|c|c|c|}
\hline \multirow[t]{2}{*}{ Patient } & \multicolumn{2}{|c|}{ Common peroneal $n$} & \multicolumn{2}{|l|}{ Tibial $n$} \\
\hline & $T L(m / s)$ & $\begin{array}{l}M C V \\
(\mathrm{~m} / \mathrm{s})\end{array}$ & $T L(m / s)$ & $\begin{array}{l}M C V \\
(\mathrm{~m} / \mathrm{s})\end{array}$ \\
\hline $\mathrm{C}$ & 0 & 0 & $7 \cdot 4$ & - \\
\hline K & 0 & 0 & $7 \cdot 6$ & 25 \\
\hline $\mathbf{L}$ & $4 \cdot 7$ & 30 & - & - \\
\hline $\mathbf{P}$ & $5 \cdot 3$ & 38 & $7 \cdot 4$ & 34 \\
\hline Q & $7 \cdot 0$ & 25 & $7 \cdot 7$ & - \\
\hline $\mathrm{T}$ & $3 \cdot 7$ & 54 & $3 \cdot 9$ & 52 \\
\hline
\end{tabular}

vary and depend on the precise composition.

Acute paralysis of distal limb muscles preceded by calf pains, subsequent development of pyramidal tract signs, and minimal sensory disturbances which were the cardinal manifestations of the present outbreak are well recognised features of delayed neurotoxicity caused by $\mathrm{TOCP}^{3}$ and also by other organophosphate (OP) compounds. ${ }^{4}$ However, certain differences were apparent in clinical features and in the outcome of the illness in the present series.

The delay between poisoning and the onset of symptoms, in case of TOCP, has been reported to vary from 8-12 days ${ }^{5}$ to $7-21$ days. $^{6}$ The delay in onset observed in the present outbreak (14-30 days, mean 25 days) could probably have been due to administration of the toxic agent in small, repeated doses over a two week period; the latent period is known to depend on the size of the dose and the length of exposure ${ }^{3}$ On the other hand, the development of paralysis in the present series had been very rapid (1-3 days), although the progress is thought to be somewhat more protracted when repeated small doses were taken. ${ }^{5}$ The duration of progress of the limb weakness in TOCP poisoning as reported by previous authors varies from a week or more ${ }^{5}$ to about two months. ${ }^{2}$

The incidence of pyramidal tract dysfunction had also been variable in the previous outbreaks. In the Moroccan episode only about $5 \%$ had definite evidence of an upper motor neurone lesion, ${ }^{7}$ whereas Vora et $a l^{8}$ found exaggerated biceps and knee reflexes in many patients affected by TOCP poisoning in Bombay. In the present series, nearly $50 \%$ of patients had some evidence of pyramidal tract dysfunction although the signs were relatively mild.

Sensory abnormalities were found only in three patients, despite careful examination. Even symptomatically, only one patient admitted to any sensory disturbances. None of the patients experienced the peripheral vascular phenomena described by Aring. ${ }^{2}$

Neurophysiological studies of OP neuropathy in man are limited and variable results have been re- ported. ${ }^{3}$ The cardinal findings of the present study were widespread neuropathic changes in the distal muscles and prolongation of TLs with relatively mild slowing of MCVs. These findings are in accordance with the results obtained in animal experiments ${ }^{9}$ and they support the current view that the neuropathy produced by OP compounds is a distal axonopathy. The signs of denervation were maximum around the eighth week after poisoning. It is interesting to note that this has been a constant finding in yet another group of patients who developed neuropathy after exposure to an OP insecticide (Senanayake, 1980, unpublished data).

Recovery from TOCP neuropathy is considered to be generally poor ${ }^{3}{ }^{6}$ In more than 100 patients seen by Aring ${ }^{2}$ 10-12 years after poisoning, spasticity of legs, foot drop and muscle wasting were still a feature. In the Moroccan epidemic, only $10 \%$ of the patients recovered completely. ${ }^{10}$ By contrast, in the present series, all the patients seen one year after poisoning were asymptomatic, despite having had complete paralysis of certain muscle groups during the early part of the illness. The degree of pyramidal tract damage had been a major determinant of the ultimate prognosis for functional recovery in previous episodes. When considered the relatively mild nature of pyramidal tract involvement, perhaps it is not surprising that our patients made a good recovery. However, it is possible that several other factors such as the age of the patients, the difference in the chemical structure of the cresyl phosphate and the duration of initial intoxication had also in some way contributed towards the favourable outcome.

Technical assistance of $\mathrm{Mr}$ RAD Nicholas, Department of Physiology, Faculty of Medicine. Peradeniya is gratefully acknowledged.

\section{References}

${ }^{1}$ Senanayake N, Jeyaratnam J. Toxic polyneuropathy due to gingili oil contaminated with tri-cresyl phosphate affecting adolescent girls in Sri Lanka. Lancet $1981 ; 1: 88-9$.

${ }^{2}$ Aring CD. The systemic affinity of triorthocresyl phosphate (Jamaica ginger palsy). Brain 1942;65: 34-47.

${ }^{3}$ Davis CS, Richardson RJ. Organophosphorus compounds. In: Spencer PS, Schaumburg $\mathrm{HH}$, eds. Experimental and Clinical Neurotoxicology. Baltimore: Williams and Wilkins, 1980:527-44.

${ }^{4}$ Hierons R, Johnson MK. Clinical and toxicological investigations of a case of delayed neuropathy in man after acute poisoning by an organophosphorus pesticide. Archives of Toxicology 1978;40:279-84.

"Cavanagh JB, Koller WC. Tri-ortho cresyl poisoning. In: Vinken PJ, Bruyn GW, eds. Handbook of 
Clinical Neurology. Vol 37. Intoxications of the Nervous System, Part 2. Amsterdam: NorthHolland Publishing Company, 1979:471-7.

${ }^{6}$ Hopkins A. Toxic neuropathy due to industrial agents. In: Dyck PJ, Thomas PK, Lambert EH, eds. Peripheral Neuropathy. Philadelphia: Saunders, 12115.

7 Travers PR. The result of intoxication with orthocresyl phosphate absorbed from contaminated cooking oil, as seen in 4029 patients in Morocco. Proceedings of the Royal Society of Medicine 1962; $55: 57-60$.
${ }^{8}$ Vora DD, Dastur DK, Braganca BM, Parihar LM, Iyer CGS, Fondekar RB, Prabhakaran K. Toxic polyneuritis in Bombay due to ortho-cresyl-phosphate poisoning. Journal of Neurology, Neurosurgery and Psychiatry 1962;25:234-42.

${ }^{9}$ Hern JEC. Tri-ortho-cresyl phosphate neuropathy in the Baboon. In: Desmedt JE, ed. New developments in Electromyography and Clinical Neurophysiology. Vol 2. Basel: Karger, 1973:181-7.

${ }^{10}$ Svennilson E. Studies of triorthocresyl phosphate neuropathy, Morocco 1960. Acta Psychiatrica Scandinavica 1961 ;suppl 150, 36:334-6. 